\title{
Primate head and body restraint without chronic skin openings or attachments to the animal
}

\author{
DANIEL KURTZ \\ New England College of Optometry, Boston, Massachusetts \\ and \\ D. MAX SNODDERLY \\ Eye Research Institute of Retina Foundation and Harvard Medical School, Boston, Massachusetts
}

\begin{abstract}
We developed a primate restraint system that requires no chronic skin openings or attachments to the animal. The restraining chair has a unique neck clasp; monkeys without chains and collars are easily trained to readily enter the chair and accept restraint with the neck and head held at a comfortable angle. A bite bar, in combination with contact on broad areas of the monkey's brow and occiput, provides rigid head immobilization. In order to achieve contact with a broad area of the occipital bone, the muscles at the back of the animal's head are surgically detached from the occiput and reattached to the underlying neck muscles. A strain-gauge, mounted on the head-holder and monitored by a laboratory computer, detects head movements of the monkey and permits the experimenter to teach the monkey to sit still during data acquisition. This system is well accepted by experienced monkeys and helps prevent the risks of infection posed by most earlier methods. Furthermore, the head and shoulders of the monkey are readily accessible for examination and for close positioning of test equipment.
\end{abstract}

Many neurophysiological and behavioral studies require head immobilization of awake monkeys (Evarts, 1968; Friendlich, 1973; Moody, Stebbins, \& Miller, 1970). Most restraint systems that achieve rigid head immobilization entail chronic skin openings, which lead to infections and difficulties in maintaining the health of the subject for long-term experiments.

In this paper we describe a rigid head restraint system which does not require chronic skin openings and which involves a minimum of hardware. The low bulk of the system leaves the monkey's head and shoulders accessible to manipulation, and the animal can be placed within a few centimeters of test equipment.

Primate head restraint systems usually require body restraint as well to absorb the force of the animal's movements. Several different designs for body restraints, or monkey chairs, have been described (e.g., Glassman, Negrao, \& Doty, 1969; Lilly, 1958; Moody et al., 1970; Nakamura, Coates, Crawford, \& Friedman, 1982). When

We thank Robert F. Thompson and Dr, George T. Timberlake for assistance with the design of the restraint system and George Bearse for construction and modification. This research was supported at various times in part by grants from the American Heart Association (74760), the National Institute of Health (EY 01520 and T32 EY 07028), the Massachusetts Lions Eye Research Fund, the National Science Foundation (BNS 78-170804), and institutional funds of the Eye Research Institute. D. Kurtz's mailing address is: New England College of Optometry, 424 Beacon Street, Boston, MA 02115. D. M. Snodderly's mailing address is: Eye Research Institute of Retina Foundation, 20 Staniford Street, Boston, MA 02114. using these devices, the experimenter ordinarily transfers the monkey from its home cage by pulling on a chain or a pole attached to a collar worn permanently by the animal (Fielder \& Casmer, 1966; Glassman et al., 1969). Monkeys sometimes fight the collar and chain, and both the handler and the animal risk injury. Unlike previous designs, the monkey chair described here does not require handling the animal, eliminates collars and chains, and allows nontraumatic immobilization of the head.

\section{RESTRAINING CHAIR}

This monkey chair is similar to an earlier design (Glassman et al., 1969) in four ways: (1) The chair temporarily attaches to the animal's home cage; (2) the monkey enters the chair through an open back, which is then blocked off by a plastic slide (as the monkey is guided into the chair, the monkey has no opportunity to leave enclosed space); (3) the top of the chair is easily removed after the monkey's neck is restrained, facilitating the experimenter's access to the monkey's head, body, and limbs; and (4) the monkey sits on a flat array of bars that allows urine and feces to drop into a pan at the bottom of the chair. Because the height of the bar array is adjustable, the seating arrangement can be made comfortable for a variety of macaque species regardless of tail length. Cynomolgus monkeys show no ill effects from daily restraint for periods of up to $6 \mathrm{~h} /$ day.

The unique feature of our chair is the low-bulk clasp used to secure the monkey's neck (Figure 1). A narrow 


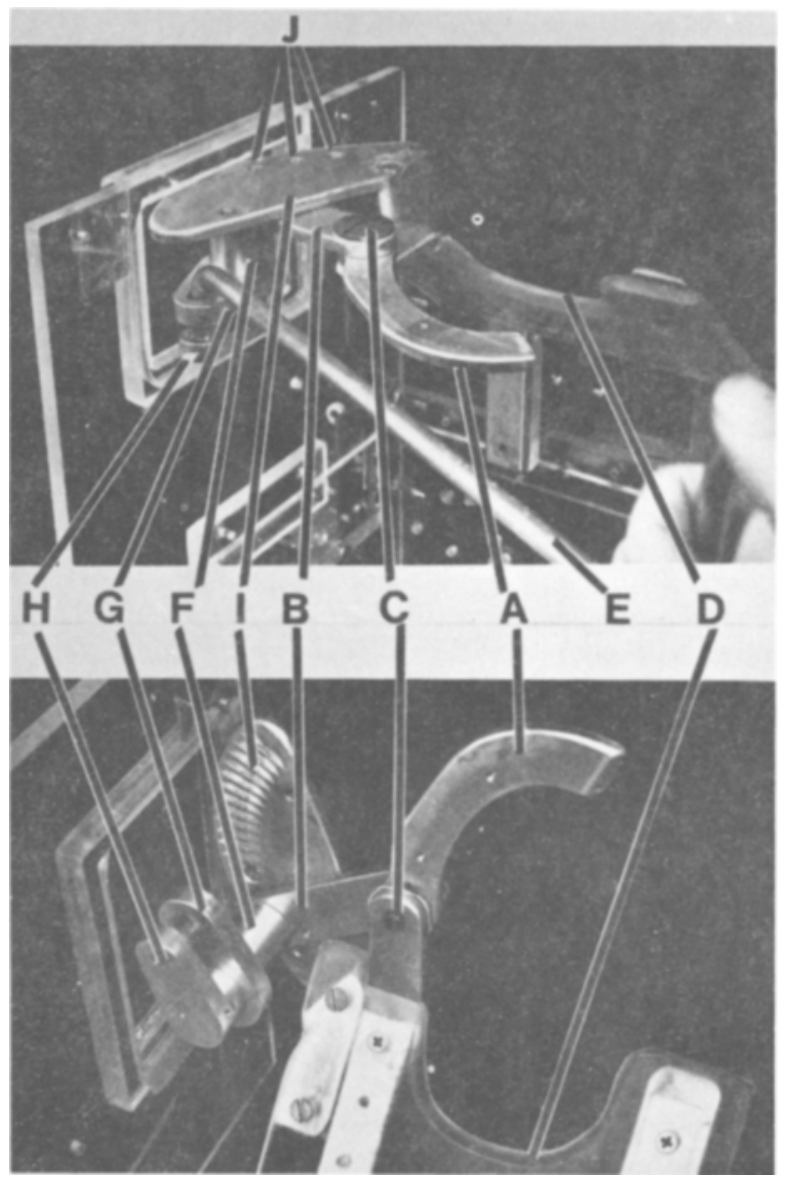

Figure 1. Low-bulk clasp used to secure monkey to the chair shown with top and back of chair removed. Upper picture: view from the back right corner of the chair. Metal arm is half-closed, control rod hooked on to pawl housing. Lower picture: view from below the neck clasp, looking up. (A) metal securing arm, (B) arm-control lever, (C) pivot point of metal securing arm, (D) plastic front piece, (E) 0.5 in. control rod, (F) pawl housing, (G) metal tab attached to pawl housing, (H) pawl release lever, (I) ratchet plate, $(J)$ holes to receive tip of pawl and lock arm open, half closed, or fully closed.

The pawl and pawl-spring, located inside the pawl housing, cannot be seen in this figure. The ratchet can be seen on the underside of the ratchet plate in the lower picture.

metal arm holds the animal's neck against a plastic front piece that is attached to the lower part of the chair and is angled downward away from the monkey at $\mathbf{4 5} \mathrm{deg}$ from the horizontal. The angle is such that the monkey can look forward while holding its head in a natural, comfortable posture (see discussion in Nakamura et al., 1982). A rounded cutout in the front plate, together with the metal arm, when closed, forms an enclosure that is larger than the monkey's neck but smaller than its head.

The motion of the capturing arm is controlled by a lever containing a spring-loaded pawl. The pawl can lock the lever to prevent the monkey from opening or closing the neck-restraining arm itself. A 0.5-in. diam control rod, which fits through a hole in the back of the chair, is used to move the lever and capturing arm while the chair is completely enclosed, ensuring the security of the mon- key at all times. At its end the rod has a 90-deg bend, which hooks onto a metal tab attached to the pawl housing. By pulling or pushing horizontally on this tab, the experimenter rotates the arm-control lever and metal arm about the pivot point. Normally the arm is locked in position by the pawl, which is held against the ratchet plate or in a locking hole by a spring inside the pawl housing. The pawl must be depressed by pushing down on the pawl release lever with the control rod to unlock the restraining arm and open or close it. This arm releases the animal from the restraint or captures it as desired. When outside the locking holes, the pawl engages a ratchet plate so that the arm can be moved forward gradually but the monkey cannot open it. The pawl and ratchet are strong enough to secure the lever and arm, which in turn keep the monkey cuffed to the chair. We place a locking screw through the lever in its closed position for additional security during lengthy experiments.

In normal operation, the chair is attached to the animal's cage, the back is removed, and the cage's guillotine door is opened. Experimenters can force recalcitrant animals into the chair by pulling forward the squeeze back of the cage. When the monkey is inside the chair, the chair back is replaced and the animal is captured. With small amounts of fruit or water the monkey is lured into position and, because the capturing arm is small, unobtrusive, and rapidly moved, the monkey is captured by the neck before it moves away. The capturing arm operates much like a handcuff. When monkeys are tested daily, they learn to enter the chair freely and to accept restraint calmly, as previously described by Barrow, Luschei, Nathan, and Saslow (1966). If the monkey does not cooperate readily, we raise the adjustable floor grid until the animal's head is in the desired position, whereupon it is quickly captured. Once the neck is restrained, a separate back piece bearing arm restraints (Figure 2) is inserted, and the monkey's arms are restrained so that one hand is positioned at a response lever. The arm restraint limits lateral movements of the thorax, but it eliminates the need for a waist restraint, and the monkey is free to move the hips and lower body at will. During the experiment, monkeys frequently make postural adjustments and they learn to do this without placing stress on the head restraint. This body freedom probably contributes to the monkey's comfort and willingness to remain placid for long periods of time.

At the end of daily testing, the monkey is freed from the arm restraint, the sliding back of the chair is replaced, and the neck cuff is opened with the control rod while the monkey is fully enclosed. The chair is then clipped back onto the cage, the guillotine cage door is raised, the sliding back is removed, and the monkey freely enters its home cage.

\section{HEAD HOLDER}

The head-immobilization apparatus incorporates three important features: (1) a bite bar and two contact pads 


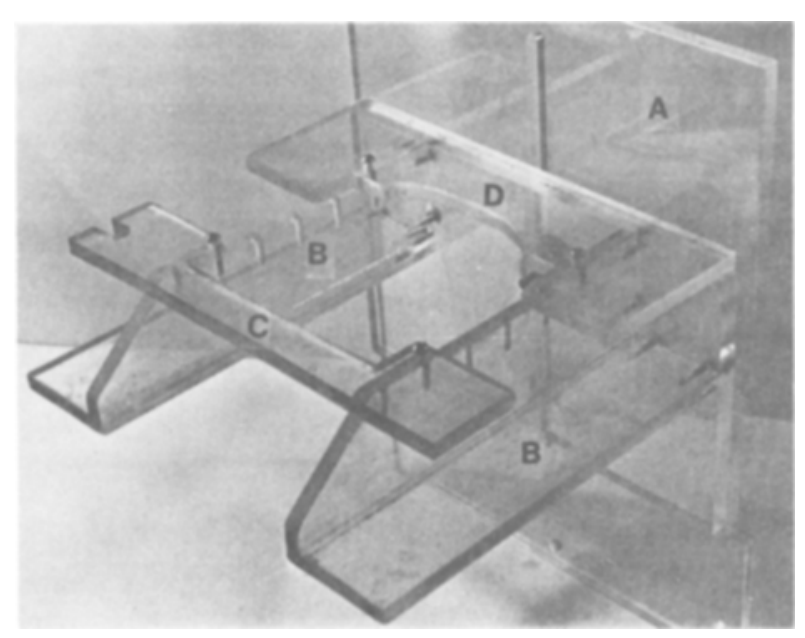

Figure 2. Removable back piece bearing arm restraints. (A) back piece, (B) channels for forearms, (C) wrist barrier to prevent elevation of forearm, (D) elbow barrier to prevent arm from being pulled backward.

When the monkey is restrained at the neck and seated comfortably in the chair, this back is inserted with the forearm channels low and the elbow barrier already in place. The channels are then raised to the height of the monkey's elbows. Finally, the wrist barrier is inserted and secured.

that restrain the skull, (2) a means of delivering liquids to the back of the monkey's mouth, and (3) a sensor to detect flexure of the headholder.

\section{Bite Bar and Contact Pads}

In our system, as in the system described by Schrier, Povar, and Vaughan (1970), the monkey's head is held at three distributed locations: the upper teeth, the brow or frontal bone, and the back of the head. The teeth are the main region of contact, and they are held by a bite bar, which is molded to the monkey's upper molar and bicuspid teeth. A stainless steel tray (Figure 3) is cut away to allow clearance of the incisor and canine teeth. Silicone rubber compound, such as is used for human orthopedic devices (Ali Med, Boston, Massachusetts), is mixed with catalyst so that it cures within 5 min or less. After the catalyst is added, the silicone is immediately placed in the metal tray and inserted into the monkey's mouth, where it cures. To open the monkey's mouth, we grasp one canine tooth with large forceps and tilt the head backward.

During the curing process, monkeys are restrained by pads on the brow and the occipital region, and by a notch in part of the reinforcement-delivery system that contacts the incisors (see below). Experienced monkeys sit still during the 5-10 min curing process, and detailed impressions of the animals' teeth are obtained. These impressions usually last several weeks.
As long as the teeth remain in firm contact with the cured silicone impression, the monkey cannot move its head in any direction relative to the steel tray. Firm contact with the dental impression is ensured by applying downward pressure on the brow and preventing the animal from rotating its head about the interaural axis by means of a contact pad at the occiput. Contact with the teeth, brow, and occiput is made via molded silicone rubber pads mounted on metal, so that only the resilient rubber actually touches the animal. The rubber pads distribute the pressure in each location over several square centimenters. Monkeys are often restrained for 5-6 $\mathrm{h}$ without ill effects and without prolonged struggling.

In order to prevent rotation of the head and withdrawal from the bite bar, minor surgery is necessary to create a recess at the posterior crest of the monkey's occipital bone (the occiput). The contact pad at the occiput fits into this recess. With the monkey under anesthesia, two layers of muscle are detached from the occipital bone and reattached to the underlying muscles, as shown schematically in Figure 4. Employing aseptic surgery, we make a
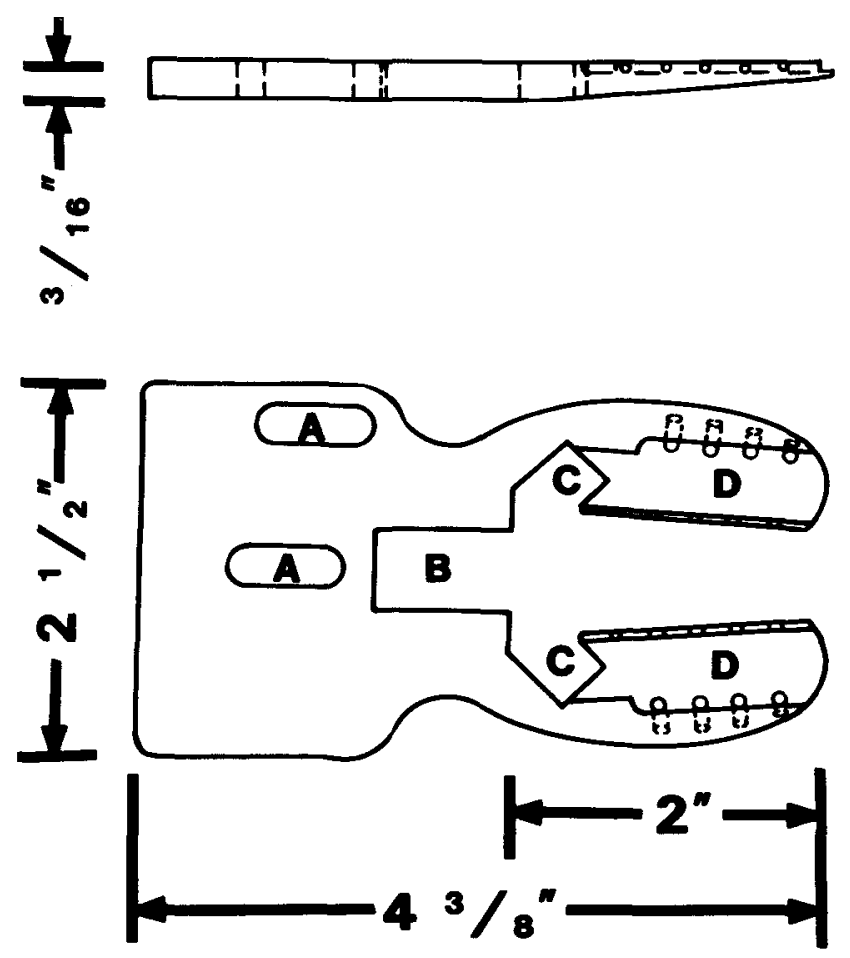

Figure 3. Scale drawing of the stainless steel tray used to hold the silicone dental impression, or bite bar. Upper panel is a side view. The outer edges are rounded to assure the animal's comfort. Spaces are cut out to accommodate (A) mounting bolts, (B) the waterdelivery goose-neck, and $(C)$ the monkey's canine teeth. The silicone molds are formed in (D) rectangular channels. Holes are drilled in the inside walls of the channels and diagonally from the outside corners of the channels to increase adherence of the cured silicone to the tray. (A detailed machinist's drawing of the tray will be sent upon request). 


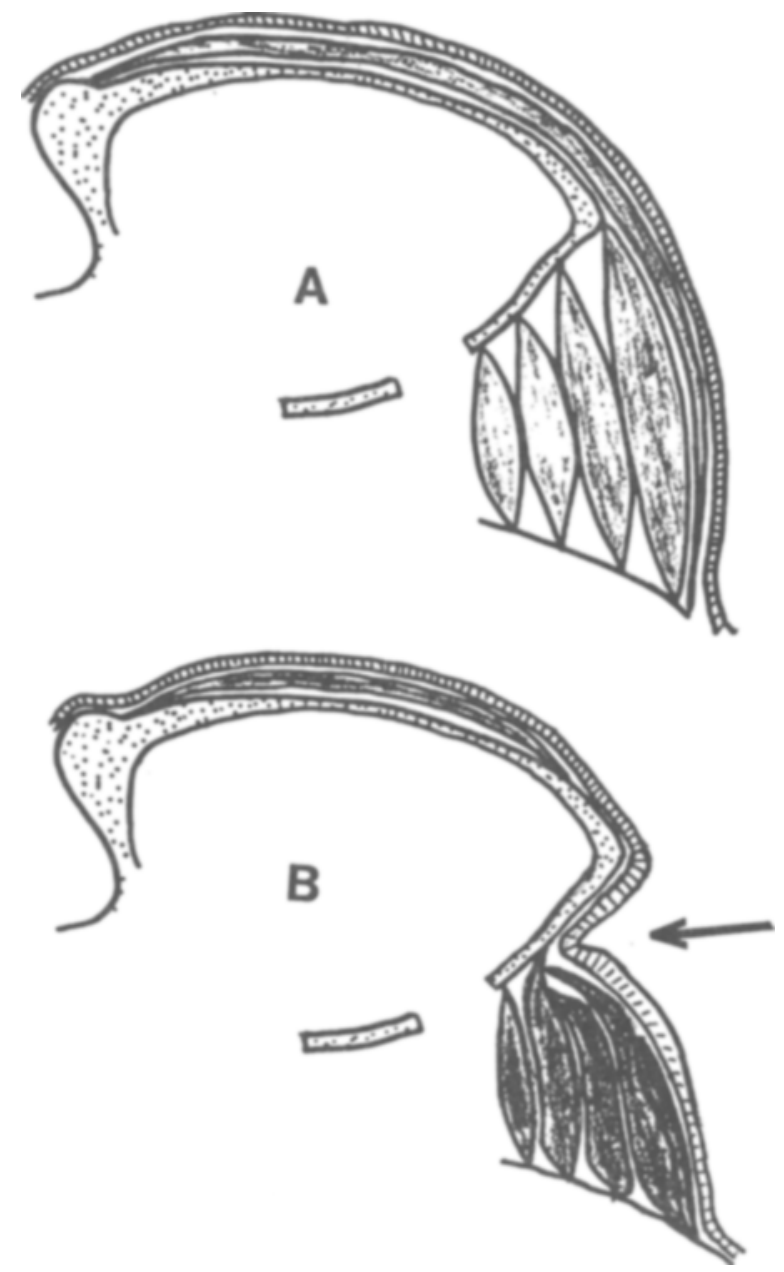

Figure 4. Schematic diagram of the skull and neck of the monkey in mid-sagittal view. Sketch $A$ represents the normal adult male monkey. Sketch B shows the effects of dissecting the occipitalis muscle and reattaching the two superficial neck muscles to the deep muscles. Note the resulting recess, formed at the back of the head (arrow). The occipital pad extended into this recess and prevented the monkey from rotating the occiput downward about the interaural axis. The arrow also shows the location of the nylon sutures used to pull the skin down onto the underlying skull and muscle during healing.

horizontal incision just above the monkey's shoulder blades. The incision is placed so that the scar subsequent to healing will be located away from the occipital contact pad. The skin is separated from the underlying fascia and retracted forward to expose the soft tissue at the back of the neck. The fascia is then cut and separated from the underlying thin muscle, the occipitalis muscle, which is detached by blunt dissection to expose the occiput. No other provisions are made for the occipitalis muscle. Then the two most dorsal layers of neck muscle are detached from the occipital bone, taking care to minimize damage to the muscles themselves. The detached ends of the superficial neck muscles are sutured with absorbable gut to the deeper, intact muscles $1-2 \mathrm{~cm}$ from the bone so that they reattach to the other muscles rather than to the bone.
The skin opening at the base of the neck is then closed, and nylon sutures are drawn between the loose skin and the underlying muscle in order to secure the skin to the bone and muscle during healing. These nylon sutures are removed 1-2 weeks postoperatively, when healing has progressed to the point at which the skin has reattached to the underlying tissue. The skin at the back of the head then follows the contours of the occipital bone. Monkeys recover quickly and uneventfully from surgery and show no obvious postoperative impairment in head movement or postural control. Contact with the back of the head, described above, is applied only after 4-6 weeks, when the skin opening is fully healed and edema has completely subsided.

The bite bar and the frontal and occipital silicone pads are attached to a metal frame (see Figure 5) that allows adjustment and locking of the contact pads. The occipital pad is affixed to a $U$-shaped bracket that slides in slots at the back of the metal mount. This bracket can be moved forward to the desired position and also pivoted to any angle to cup the animal's occiput. Stainless steel 8-32 machine screws are threaded into the U-bracket and covered with silicone rubber that is molded to conform to

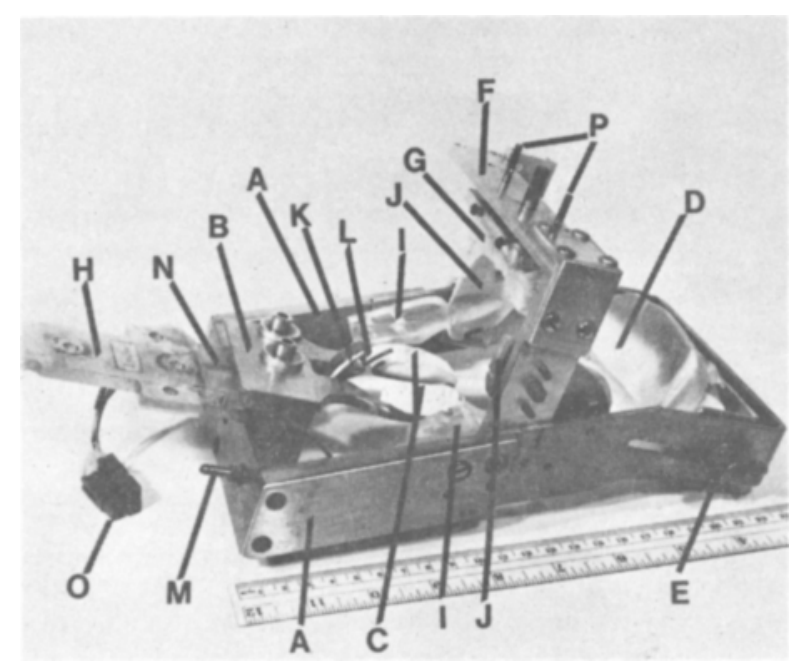

Figure 5. Rectangular head mount and associated hardware (the left side faces the front of photo): (A) main rectangular frame, (B) stainless steel tray for holding dental impressions, (C) dental impressions in stainless steel tray, (D) occipital contact pad, (E) wing nut to secure occipital pad (another wing nut is hidden from view on the other side of the frame), (F) bridgework for frontal and lateral contact pads, (G) frontal pad, (H) flat attachment plate used to mount head holder to XYZ positioner, (I) plastic barriers used early in training to restrict range of head movement, $(J)$ contact pads to restrain lateral movements used early in training, $(K)$ steel notch for incisors on end of stalk, (L) polyethylene tubing for liquid reinforcement partially extended beyond lower jaw of the notch, $(M)$ liquid input nipple for tubing from peristaltic pump, (N) strain gauge, covered by acrylic plate for protection, $(O)$ connector between strain gauge and amplifying circuitry, $(P)$ guide rods. 
the back of the head. The occipital bracket is locked in place by wing nuts on each side of the rectangular frame.

The frontal pad is supported by a stationary vertical bridge that spans the horizontal antero-posterior frame. The top span has clearance holes for rods that are attached to a metal plate with the molded silicone rubber pad. The large center rod can be locked with a set screw at any vertical position; the two smaller guide rods prevent the pad from rotating while it slides into the appropriate vertical position. Similar arrangements on the vertical sides of the bridgework permit lateral restraint of the head by two additional pads that are used during the early phases of training.

The entire frame is attached in front to a flat aluminum plate that can be bolted to a table or mechanical positioner. When the attachment plate is mounted horizontally, the front part of the antero-posterior rectangular frame angles downward at $24 \mathrm{deg}$. The back part of the frame, which guides the occipital pad, is then parallel to the attachment plate (i.e., horizontal). Thus, when the monkey is restrained, most of the upper part of its head is accessible and its visual field is unrestricted in all directions except inferiorly (see Figure 6). [McNamara (1973) described a head restraint system which grasped the monkey's head at the occiput and at the brow but not at the teeth. This system was very bulky and obscured the eyes. For this reason, it is not suitable for visual or oculomotor research; in addition, no measure of the stability of the monkey's head was provided.]

Early in the monkey's training, plastic barriers and lateral contact pads are placed inside the frame to limit the monkey's head movements while the bite bar is inserted. Experienced monkeys cooperate with insertion of the bite bar, so these barriers can be removed. In addition, early in training, lateral contact pads help to reduce the monkey's movements while the dental impression is hardening. These pads, which make contact just anterior to each ear, are unnecessary for experienced subjects and are eventually removed.

\section{Reinforcement Delivery}

By delivering liquid to the back of the monkey's mouth, the reinforcement system enables the animal to swallow even though the mouth is held open slightly by the bite bar and the incisor rest. The contact point of the reinforcement system is a stainless steel notch on the end of a stalk, which resembles the neck and head of a goose with its mouth open (see Figure 5). The incisors rest on the lower fork of this notch during the curing of the dental impression. The stalk provides clearance between the anteroposterior metal frame and the animal's mandible. The lower fork of the notch has a clearance hole through which polyethylene tubing is passed (PE 200, o.d. 0.075 in.). The tubing is withdrawn into the stalk so that it is out of the way while the monkey is being positioned in the frame.

After the monkey is fully restrained, the tubing is pushed through the incisor rest until it is past the hump of the tongue. Liquid reinforcement is pumped through
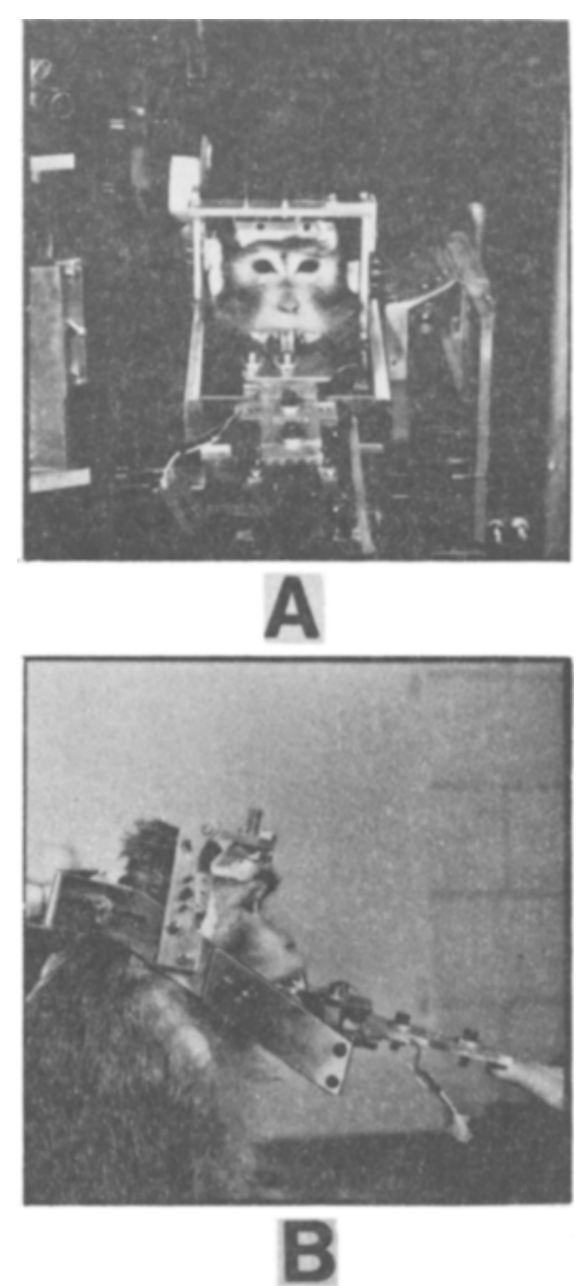

Figure 6. Views of a monkey restrained by the headholder. (A) Frontal view with monkey seated in chair adjacent to experimen-

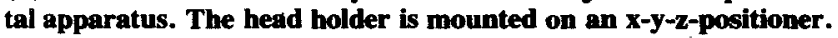
Notice the closeness of the monkey to the equipment at the left of the picture. (B) Side view. Headholder detached from apparatus.

this tubing by a Masterflex tubing pump (Cole Parmer, Chicago, Illinois) driven by a stepping motor under computer control.

\section{Strain Gauge}

A strain gauge (Type FAE-25-35-S6ET, BLH Electronics, Waltham, Massachusetts), a resistor that changes its value when flexed, is cemented to the flat metal attachment plate and is covered by an acrylic plate for protection. A weak point is created in the attachment plate by drilling a hole through its width at the location of the strain gauge. This weak point, and the strain gauge, are thus the first parts of the restraint system to flex when the animal moves. Resistance changes due to flexure of the strain gauge are converted to voltage changes by a Wheatstone bridge circuit. The voltage signal is amplified further by an amplifier with a low frequency cutoff of $0.1 \mathrm{~Hz}$ to eliminate dc drifts. A computer analog in- 
put samples the amplifier output. Whenever the strain gauge signal exceeds a preset threshold, the animal is punished by receiving a timeout so that the next opportunity for reinforcement is delayed. A repetitive beep is also sounded by a Sonalert as an auditory cue during the time when the strain threshold is exceeded. By gradually reducing the threshold voltage, we train the monkeys to sit still most of the time.

When, despite its training, a monkey moves its head, the computer detects the movement, disregards the potentially erroneous data, and again delays the opportunity for reinforcement. The system is sensitive to head displacement of as little as $0.2 \mathrm{~mm}$, a degree of freedom we believe to be smaller than that tolerated by other headrestraint systems. The residual displacements allowed by other systems of head restraint have not been reported.

Monkeys restrained daily by this system have remained vigorous and healthy for over two years with no apparent ill effects.

\section{ADVANTAGES AND DISADVANTAGES OF THE SYSTEM}

We had to satisfy two major desiderata in designing this system: (1) The monkey's head had to be placed near an eyetracking device that had been designed for human subjects (Snodderly \& Kurtz, 1985), and (2) we wanted to conduct long-term behavioral training to compare monkeys' performance on visual tasks with that of human subjects tested under identical conditions. Thus it was important that the monkeys remain healthy and perform stably for long periods of time.

Existing techniques were unsuitable because all of the monkey chairs previously described enclosed the monkey's shoulders in a bulky plastic box that placed the animal too far from the apparatus. Although our design solves this problem for apparatus brought from the right side, access from the left side still is partially obstructed (Figure 6).

The advantages of the head restraint are threefold: (1) There is no risk of infection; (2) the monkey can be taught to sit still, eliminating movement artifacts from the measurements; and (3) the location of the head and eyes can be adjusted precisely to specified coordinates by an external positioner. A closed circuit television monitor is used to align the monkey's eye to the same position each day.

The basic design of the headholder can be modified to meet many different requirements. For example, the vertical bridgework can be moved forward or backward to favor access to the eyes or the ears. The range of adjustment should be adequate to accommodate different sizes of monkeys, and it is likely that monkeys could be retrained at repeated intervals during the life cycle for be havioral testing with this or a similar system.

Our approach has three major disadvantages. First, when the monkey is restrained each day, the head is in a slightly different position from the previous day. If a new dental mold must be made, the position of the head must be adjusted to bring the eye, for example, to the same location in space each day. This daily readjustment of head position is often unnecessary for animals with rigid chronic implants, as long as the positioning is not hypercritical.

Second, the restraint of the head takes some experimental time because the bite bar must be inserted and the positions of the contact pads must be adjusted each day. Head restraint is probably faster with rigid skull implants.

Finally, and most importantly, the length of time that the monkey can be restrained with our technique is limited. When the monkey is first restrained, he will sit and work quietly at the behaviorai task for long periods of time. As the day proceeds, bouts of fidgeting become more frequent and performance eventually declines. For this reason we usually have not tried to restrain the animal for longer than $6 \mathrm{~h}$ at a time. Some of the fidgeting may be due to the desire of the animal to change body or head position. Interrupting the experiment and freeing the animal's head for brief periods at regular intervals might make it possible to extend data gathering or training for more hours per day.

\section{REFERENCES}

Barrow, S., Luschei, E., Nathan, M., \& Saslow, C. (1966). A training technique for the daily chairing of monkeys. Journal of the Experimental Analysis of Behavior, 9, 680 .

EVARTS, E. V. (1968). A technique for recording activity of subcortical neurons in moving animals. Encephalography \& Clinical Neurophysiology, 24, 83-86.

Fielder, F. G., \& CASMER, C. J. (1966). A device to facilitate the restraint and handling of monkeys with minimal human contact. Laboratory Animal Care, 16, 395-402.

FriendLICH, A. R. (1973). Primate head restrainer using a non-surgical technique. Journal of Applied Physiology, 35, 934-935.

Glassman, R. B., Negrao, N., \& Doty, R. W. (1969). A safe and reliable method for temporary restraint of monkeys. Physiology \& Behavior, 4, 431-432.

LiLLY, J. C. (1958). Development of a double-table chair method of restraining monkeys for physiological and psychological research. Journal of Applied Physiology, 12, 134-136.

McNamarA, J. A., JR. (1973). Restraint of monkeys for craniofacial research. Journal of Dental Research, 52, 183.

Moody, D. B., Stebins, W. C., \& Miller, J. M. (1970). A primate restraint and handling system for auditory research. Behavior Research Methods \& Instrumentation, 2, 180-182.

Nakamura, R. K., Coates, R., Crawford, H., \& Friedman, D. (1982). A flexible restraint chair for the cynomolgus monkey (Macaca facicularis). Journal of Medical Primatology, 11, 178-185.

Schrier, A. M., Povar, M. L., \& Vaughan, J. (1970). Measurement of eye orientation of monkeys during visual discrimination. Behavior Research Methods \& Instrumentation, 2, 55-62.

SNODDERLY, D. M., \& KURTZ, D. (1985). Eye position during fixation tasks: Comparison of macaque and human. Vision Research, 25 (1), 83-98.

(Manuscript received December 4, 1984; revision accepted for publication April 20, 1985.) 